\title{
Introducing a Workshop to Build an Affordable 3D-Scanner: Presenting a Variety of Computational Concepts to Novice Students of Architecture
}

\author{
Werner Lonsing \\ Independet Researcher, Germany \\ sigradi2013@lonsing.com
}

\begin{abstract}
This paper outlines and describes a workshop designed for presenting and teaching a variety of computational concepts to novice students of architecture. While the ostensible purpose is the building of a cheap scanner the workshop facilitates a variety of techniques like model-making, basics of robotics or 3D-point-clouds. Novice students can discover a broad range of computational technologies both in hardware and software over a short period of time.
\end{abstract}

Keywords: FabScan; Workshop; 3D-Scanner; Arduino; point-cloud.

\section{Introduction}

Teaching architectural design is to some extent an exception in education, because building models and drawing skteches are actions to create fact physical representations, and some seminars and studios may in fact resemble the appearance of a workshop. It is more than just the transfer of knowledge. We all know about it.

By contrats teaching computation in architectural design, especially for novice students, very often simply boils down to frontal instruction, because it is only the transfer of knowledge. There are no real alternatives to talk about how to draw shapes on a computer. With a second front in place, the front of the computer, which always diverts attention, these classes are not for fun for both students and teachers.

\section{Workshops}

Student workshops can deliver an intensive experience over a short period of time. Their success depend very much on the novelty and diversification of the subject to arouse some interests. If only some nice memories are collected the results by educational means are a flash in the pan and can be neglected. They always have to be new and interesting.

A first attempt to use this pattern in order to break the unsatisfactional teaching of computational design was to organize a workshop to build a low-cost 3D-printer. Very soon it turned out, that these machine are too complex for starters. RepRaps (short for replicating rapid prototyp printer), although designed as DIYkits require some decent electrical skills like soldering SMDs. Additionally their combined parts are too expensive. As tempting a project of building such a printer still is, it is not a project for workshops or classes at architectural schools, at least not now in undergraduate education.

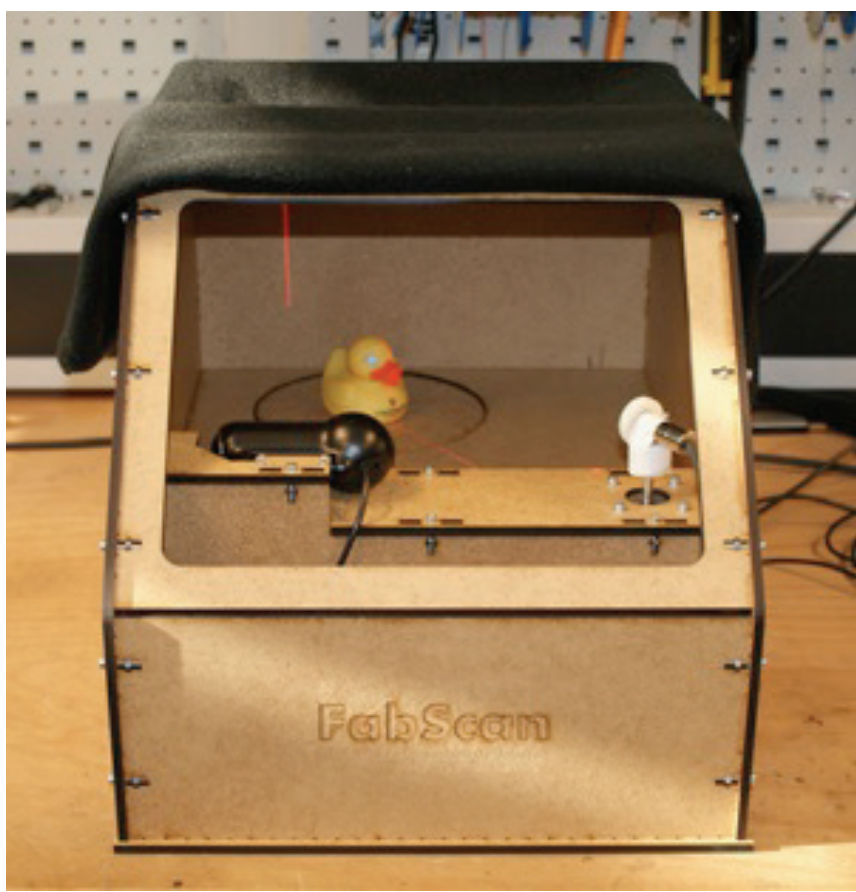

Figure 1: FabScan

\section{Introducing 'FabScan'}

Then a project named 'FabScan' showed up. It is an affordable 3Dlaser scanner developed at the Media Computing Group at RWTH Aachen University as part of a bachelor thesis. It is completely made public as an open source project. This project was used to plan a workshop for architectural students at Stuttgart university.

\section{Technical Description}

The main components of the FabScan are a webcam, a line laser, an Arduino-board, stepper-motors and an enclosure. The 
enclosure is made of laser cut MDF or plywood to be assembled with some screws. It provides space and fixing points for all other parts. The most complicated assembling is the rotating round turntable for the objects (max. $20 \mathrm{~cm} \times 20 \mathrm{~cm} \times 16 \mathrm{~cm}$ ) to be scanned. This plate is mounted on a stepper-motor, as is the line laser.

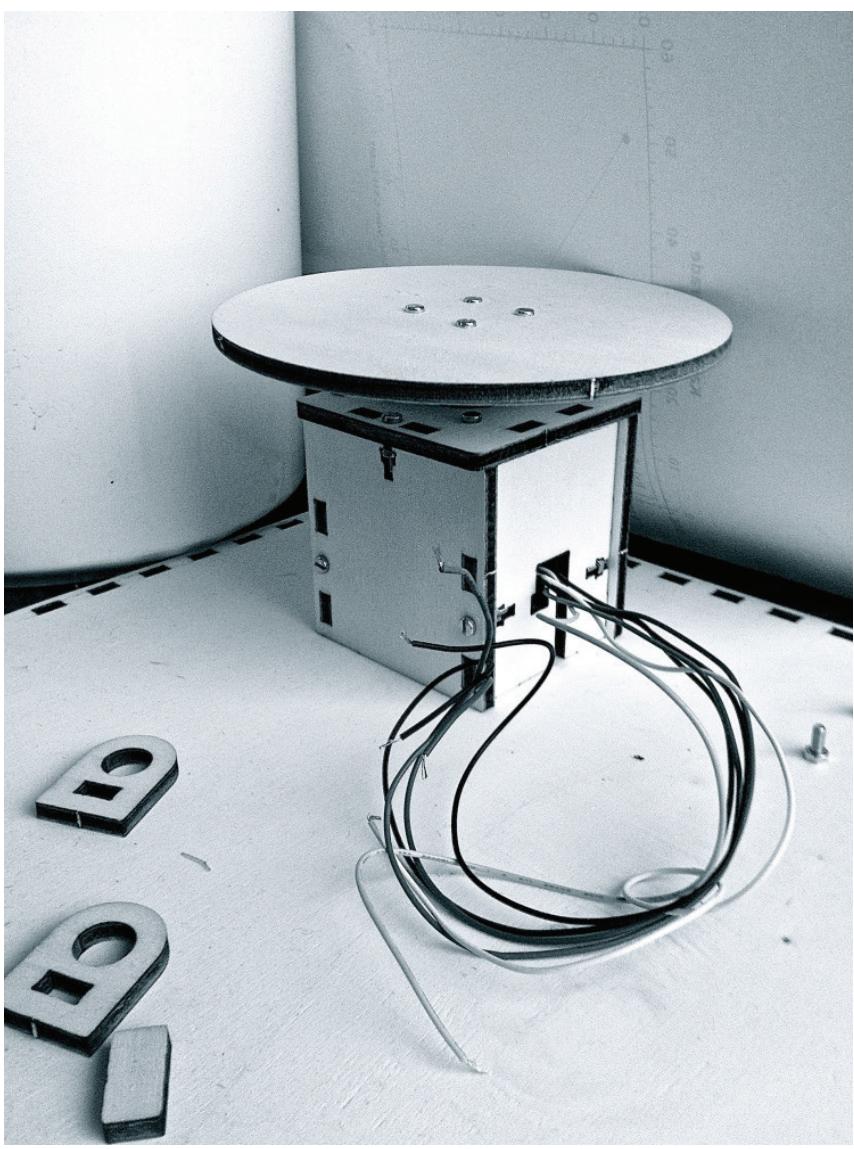

Figure 2: Assembly of the Turntable

The camera is mounted at a fixed point in juxtaposition to the turntable. As result, the complete geometry of the scene is known: axis of the turntable and both the positions of line-laser and camera. Camera and Arduino-board are then connected to a computer.

\section{History}

The first prototype was developed as thesis at the RWTH Aachen, where it was presented at an exhibition. Since then the project got its attention. It was presented in some magazines [not cited here], different models were developed and some workshops on makerfaires and also in schools have taken place.

\section{Hardware}

The hardware is comprised of the enclosure, the micro controller and peripherals. While the enclosure is custom built all the electronic parts are off-the-shelf.

\section{Enclosure}

The enclosing box is made out of laser-cutted wooden shingles. Assembly is simple and straightforward by a well prepared slot and tab construction and some screws for fixation. The plans and the layout are provided as open sourced data.

\section{Micro-controller}

The core of the FabScan is its micor-controller. A micro-controller is a small computer on a single integrated circuit. It contains a processor, some memory and a programmable interface to connect to some peripherals for input or output. Here the controller is an Arduino-board.
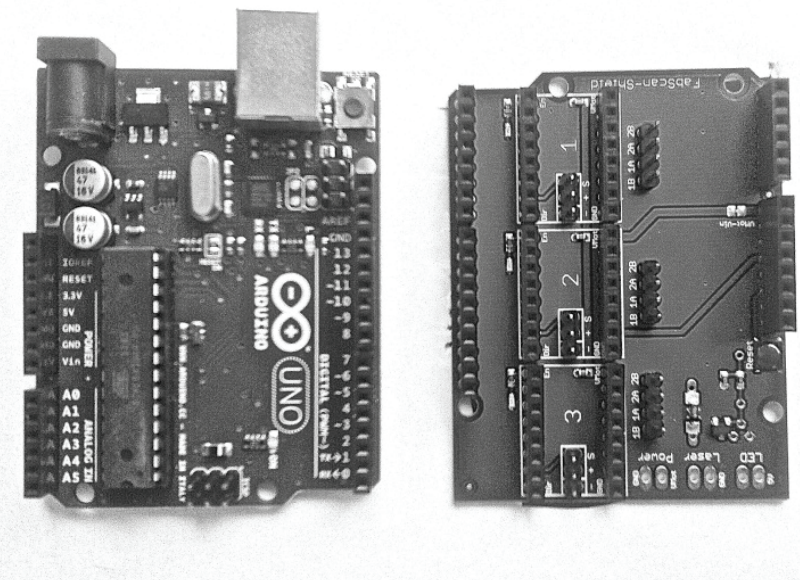

Figure 3: Arduino-board and FabScan-Shield

A special FabScan-shield is designed as an Arduino plug-on module for 3D laser scanning and already on sale. It has most of the SMD components assembled and all connectors are included. Connecting the line laser and the driver for the stepper-motors still require some electrical skills or some assistance. If desired, some LED can be applied for informational lighting.

\section{Peripherals}

Finally some peripheral devices arre added to interact with the physical environment, the object to be scannen. A laser proviodes a calibrated line on the object, a camera takes pictures and two motors adjust the laser and the turntable with the object on top of it. 


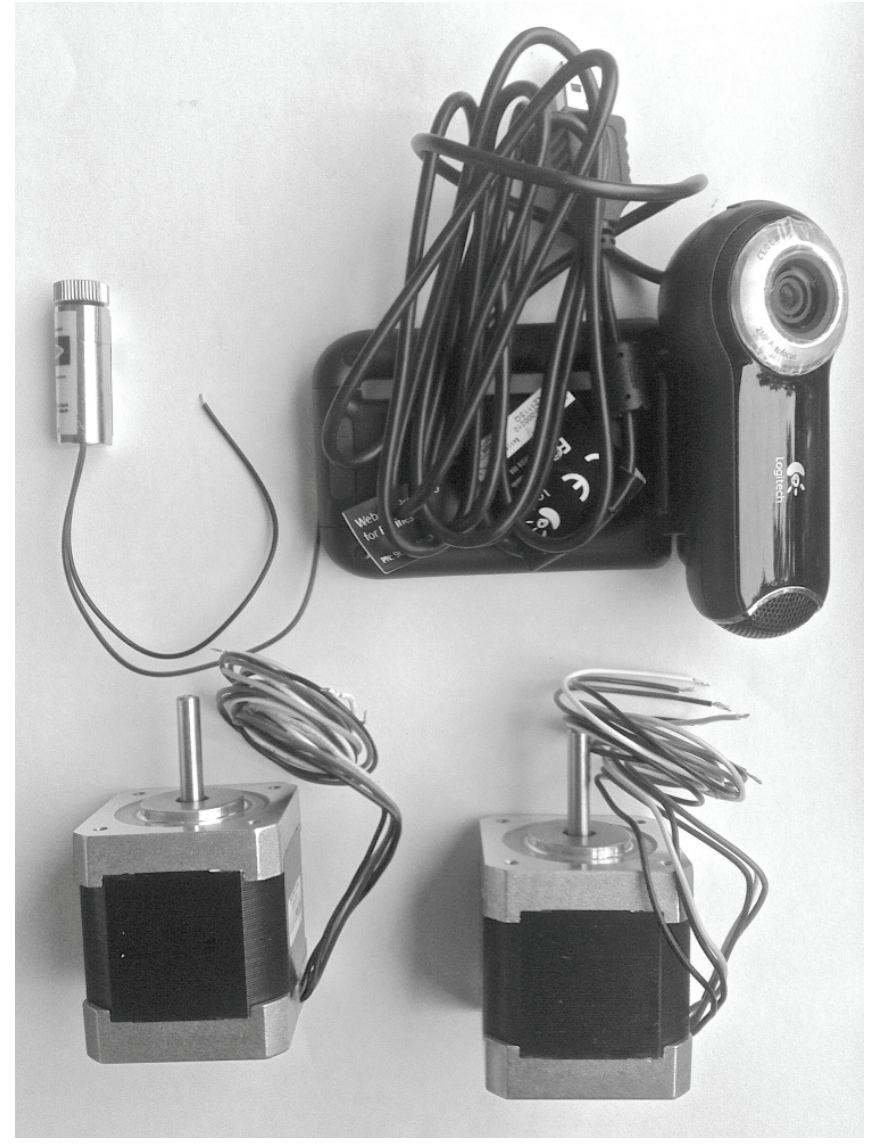

Figure 4: Laser, Camera and 2 Servo-Motors

\section{Computer}

Besides the FabScan a computer is still necessary. While microcontroller can run small and specialized applications, operations requesting more computational power or a dedicated userinterface like a keyboard or a screen are best handled with a computer as second device. In addition a this computer can be used as development environment to develop and upload the software to the micro-controller.

\section{Software}

The software is divided into two parts: the software to operate the micro-controller and all its attached hardware, and the software on the main computer to access and evaluate the frames of the camera and create the point-cloud. The software is open sourced and available at the project's homepage.

\section{Micro-Controller}

All peripheral electronic components are controlled by the Arduino-board.

Some online-distributors already provide a complete kit ready to be assembled. Only the webcam has to be purchased separately.

\section{Computer}

To run and develop a software on an embedded computer is used as development environment. This is the standard Arduio-IDE and needs no further elaboration here.

More important is the software specifically developed to import the vertical scan-lines after every rotation of the turn-tables and build them up to a point-cloud and maybe a mesh. The software uses the OpenCV and PCL (Point Cloud Library). Distributions are available for Mac and Linux.

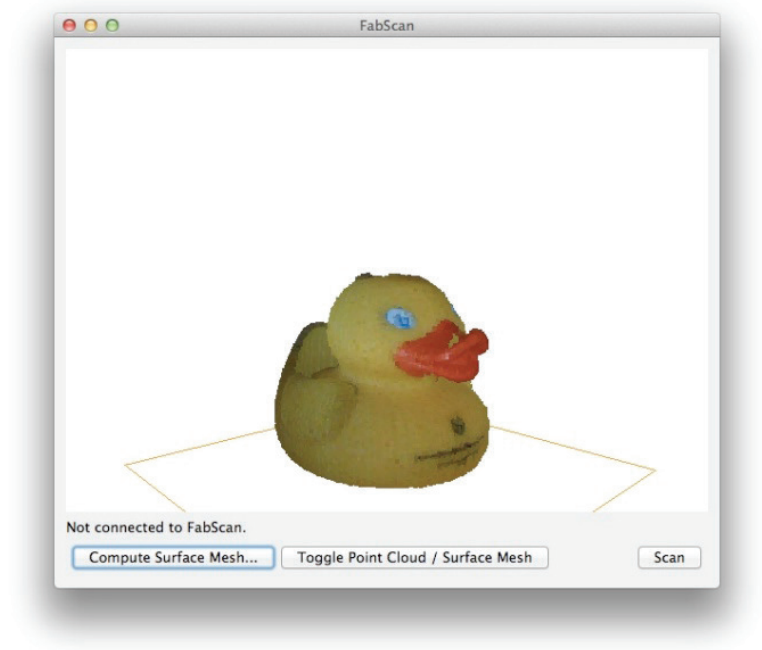

Figure 5: Software of the Scanner

\section{How it works}

The FabScan follows the principles of other 3D-scanners. A laser beam is pointed onto an object, where it is sensed with a camera. Because the laser draws a very exact line, the object can be measured. Then the camera takes a second image from the object to gain the color values of the pixels marked by the laser on the previous image, followed by a rotation of the turntable at a very small angle. After a complete fully automatic rotation by $360-$ degree the final result would be a point cloud for distribution or further manipulation. Depending on the size of the object and the desired accuracy an automated scan could take from some minutes to more than an hour.

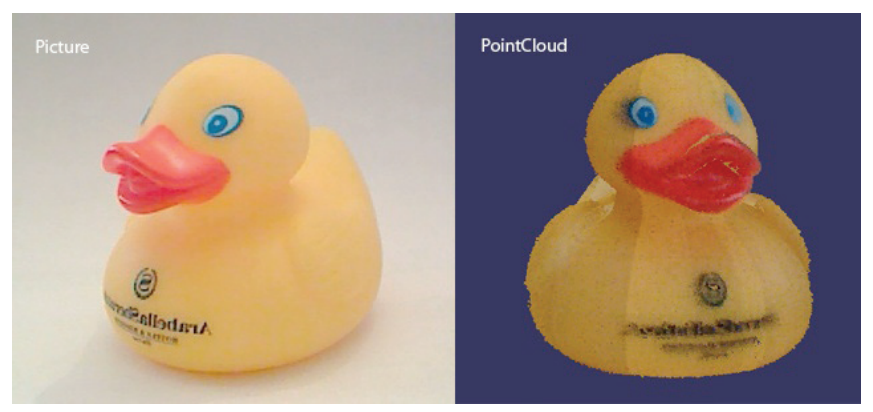

Figure 6: Object and Scan 


\section{Workshop}

The goal of this still proposed workshop is to introduce techniques of computation which are usually not subject of the architectural design curriculum. In particular this is the assembling and usage of micro-controllers in combination with peripheral devices like lasers and servo-motors. While the subject is normally not considered as suitable for architectural studies, because it is outside the common curriculum, which is usually solely based on $C A D$, a workshops gives just an impression, what technologies are at hand and, at this stage without any further requests, how they can be used. These evolving technologies are at the core of the yet emerging field of adaptive architecture.

Hence the basics of robotics with sensing and acting and some other adaptive technologies are introduced in this workshop, while the students are focussed on the main goal, building the scanner. In addition students get some computaional practice like in general dealing with open source project, installing libraries or simple tweaking of parameters.

In details these technologies are described by the workflow of the workshop. Namely these are:

1. working with a laser cutter to

2. building the enclosure and mechanics

3. assembly of electronic parts

4. install and run a program on an micro-controller

5. install a bundle of open source software on a computer

6. get a 3D-scanner (if paid for the parts)

In addition follow-up-courses can be formulated with a 3D-scanner as prerequisite. Intermingling physical and digital representations of architecture may lead to studies in a yet unknown form.

\section{Conclusion}

\section{References}

Angelo, Alex; Neves, Heloisa; de Campos, Paulo Eduardo Fonseca (2012) Fab Lab Kids: Oficina de projetos socioambientais para crianças de escolas públicas fazendo uso da eletrônica e da fabricação digital [Fab Lab Kids: Workshop for environmental projects public school children making use of electronics and digital fabrication], SIGraDi 2012 [Proceedings of the 16th Iberoamerican Congress of Digital Graphics] Brasil - Fortaleza

Borchers, Jan: Arduino in a Nutshell, 1.8, Aachen 2013: online at: The Media Computing Group : Arduino in a Nutshell. Retrieved from: http://hci.rwth-aachen.de/arduino

Braumann, Johannes and Sigrid Brell-Cokcan (2012) Digital and physical computing for industrial robots in architecture: Interfacing Arduino with industrial robots, Proceedings of the 17th International Conference on Computer Aided Architectural Design Research in Asia

Celani, Gabriela; Carlos Eduardo Verzola Vaz (2012) CAD Scripting and Visual Programming Languages for Implementing Computational Design Concepts: A Comparison from a Pedagogical Point of View, International Journal of Architectural Computing vol. 10 - no. 1, 121138
Building a functional computational device in a workshop is besides its beneficial side effects a real opportunity to teach on themes that traverse computation and architecture. In this project the programming of the micro-controller can directly interfere with the 3D-model derived from the point-cloud. The project with the familiar component of the wooden enclosure provides an insight to some very useful technologies like the usage of microcontrollers, which otherwise would be hard to present. It would be either only theoretical or a highly specialized project, where such boards are injected and became parts of some building.

The final conclusion is quite simple. Celani and Vaz (2012) in their introduction wrote: "The insertion of new technologies in architectural education has been the target of many discussions and publications, and the theme of many conferences, especially in the 1980's and 1990's, when computers became accessible for most architecture schools."(p.122) This statement could be simply repeated here.

There are two observations, though. Beyond an initial insertion with the introduction of computers the adaption of new technologies continues and for the foreseeable future will continue. There are no doubts about it. The other point is, that, coming from mainframes with terminals, computation now is moving away from office computers and desktop computing towards mobile and embedded computing.

\section{Acknowledgments}

I have to thank to Francis Engelmann and his supervisors René Bohne and Jan Borchers of the Media Computing Group at the RWTH Aachen University for developing this project and making it available as open source.

Elkær, Tim Nøhr (2009) Using Computers to Aid Creativity in the Early Stages of Design - or Not!: Rehabilitating the 2D/3D physical representation in Computer-Aided-Ideation, Computation: The New Realm of Architectural Design [27th eCAADe Conference Proceedings Istanbul (Turkey), pp. 761-768

Engelmann, Francis: FabScan - Affordable 3D Laser Scanning of Physical Objects, Bachelor Thesis Project (Supervisor: René Bohne), RWTH Aachen, not yet published, project's page: http://hci.rwthaachen.de/FabScan

Hertz, Garnet (2011) Arduino Microcontrollers and The Queen's Hamlet: Utilitarian and Hedonized DIY Practices in Contemporary Electronic Culture, ACADIA 11: Integration through Computation [Proceedings of the 31st Annual Conference of the Association for Computer Aided Design in Architecture (ACADIA) Banff (Alberta)

Huang, Yinghsiu; Hsieh, Kai-Wei; Chen, Huan-Nian (2012) The Emotional Design by Combining Interactive Technologies and Imaginations, Achten, Henri; Pavlicek, Jiri; Hulin, Jaroslav; Matejdan, Dana (eds.), Digital Physicality - Proceedings of the 30th eCAADe Conference Volume 2, Czech Technical University in Prague, Faculty of Architecture (Czech Republic), pp. 361-368 